

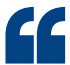

$\begin{array}{ll}\text { the pharma- } & \begin{array}{l}\text { While further work is needed, } \\ \text { the present study shows it might be } \\ \text { possible to address complex human }\end{array} \\ \text { cological } & \text { conditions such as obesity and changes } \\ \text { stimulation } & \begin{array}{l}\text { associated with ageing by targeting a } \\ \text { single receptor pathway. }\end{array}\end{array}$

markedly

increased

whole-body

energy

expenditure Receptor Signaling Ameliorates the Effects of showed increased signs of senescence and ageing in BAT and skeletal muscle, whereas, pharmacological stimulation of A2B restored much of the age-related changes."

While further work is needed, the present study shows it might be possible to address complex human associated with ageing by targeting a single receptor pathway.

Alan Morris

ORIGINAL ARTICLE Gnad, T. et al. Adenosine/A2B Aging and Counteracts Obesity. Cell Metab. https://doi.org/10.1016/j.cmet.2020.06.006 (2020) RELATED ARTICLE Batsis, J. A. \& Villareal, D. T. Sarcopenic obesity in older adults: aetiology, epidemiology and treatment strategies. Nat. Rev. Endocrinol. 14, 513-537 (2018)

Crucially, exercise training did not confer benefits in the offspring of $3^{\prime}-\mathrm{SL}$ global knockout $\left(3^{\prime}-\mathrm{SL}^{-1-}\right)$ mice. Furthermore, cross-fostering experiments showed that milk from exercised-trained $3^{\prime}-\mathrm{SL}^{-1-}$ dams did not mediate benefits on WT offspring of sedentary dams. Finally, supplementing offspring of sedentary, HFD-fed WT mice with $3^{\prime}$-SL during lactation improved their metabolism and cardiac function as adults.

"To our knowledge, this is the first study to identify a role for exercise to induce adaptations to the composition of breast milk, and also the first study to identify a role for 3'-SL to mediate improvements in metabolic health and cardiac function in offspring," concludes Stanford. "In the future, we would like to determine the mechanism through which 3 '-SL improves metabolic and cardiac function."

Shimona Starling

ORIGINAL ARTICLE Harris, J. E. et al. Exerciseinduced 3 '-sialyllactose in breast milk is a critical mediator to improve metabolic health and cardiac function in mouse offspring. Nat. Metab. https:// doi.org/10.1038/s42255-020-0223-8 (2020)
NUTRITION

\section{Towards precision nutrition}

The metabolic response to food can vary substantially from person to person, which can affect the risk of cardiometabolic disease. The factors that determine this variation in response to consuming food have been unclear; a new study published in Nature Medicine has addressed this question.

"We wanted to be able to quantify how much meal content, time of day, sleep, gut microbiome and other individual characteristics contribute to glucose levels and to develop models to describe how the interactions between individual characteristics, meal composition and other sources of variation mould postprandial glycaemia," explains author Ana Valdes. "We also wanted to compare glucose data to other important postprandial metabolic responses, specifically C-peptide and triglyceride levels." To address these questions, the researchers recruited a cohort of 1,002 healthy adults, which included a mixture of twins (to assess the genetic influence) and unrelated adults. Findings from this UK-based cohort were then validated in a cohort of 100 adults in the USA.

At baseline, the metabolic responses (blood levels of triglyceride, glucose and insulin over a 6 -h period) to a set meal were measured in the clinic.

Analysis of dried blood spots and continuous glucose monitoring (as well as a specially designed app) were used to monitor postprandial responses over a 13-day at-home period. These responses included the lipaemic response and levels of C-peptide (a surrogate for insulin) following two standard meals containing different amounts of fat and carbohydrates and the glycaemic response to eight set meals that differed in macronutrient content.

Various models were used to analyse the data, in addition to machine learning. The researchers found a large degree of interindividual variability in postprandial responses, and that these responses were fairly consistent for each individual. "This finding suggests that once we understand an individual's postprandial response to specific foods, their response to other foods can be inferred," says Valdes. "We also found that the genetic influence was less than expected, especially for triglyceride levels, while modifiable factors like meal timing conveyed larger effects than anticipated." The researchers also found that the gut microbiome composition had a considerable effect on the variance observed in postprandial lipaemia.

Valdes and colleagues have already conducted further research into the role of the gut microbiome in the findings reported in this study. "Future research is also focusing on modelling how the time of day for a meal and the amount of sleep affects postprandial responses," explains Valdes. "In addition, future research could include much more in-depth investigations of triglyceride responses, which could link glycaemic, insulinaemic and lipaemic responses to crucial pathophysiological traits, such as endothelial dysfunction."

Claire Greenhill

ORIGINAL ARTICLE Berry, S. E. et al. Human postprandial responses to food and potential for precision nutrition. Nat. Med. https://doi.org/10.1038/s41591-020-0934-0 (2020)

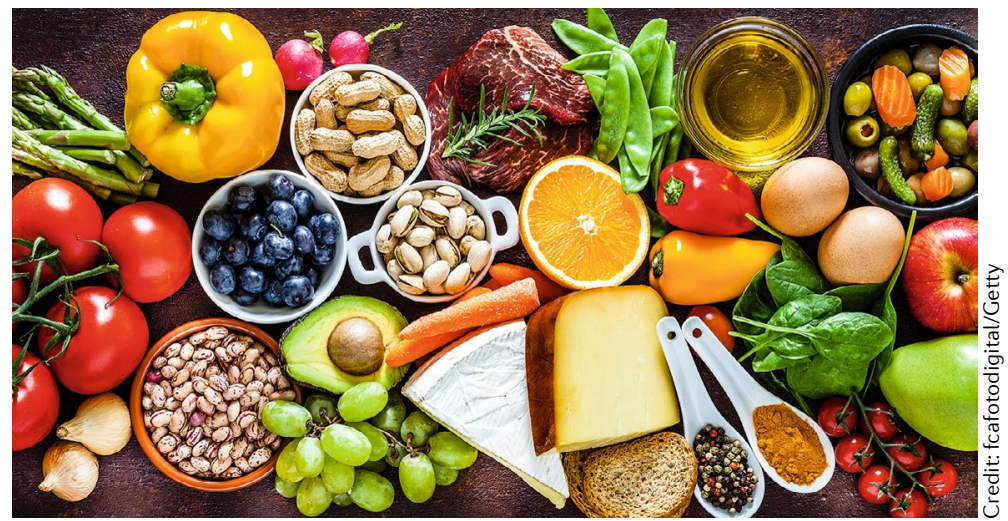

